\title{
The effect of pharmacotherapy on structural connectivity in First-Episode Drug-Naïve Adolescents with Major Depressive Disorder: 3-month prospective follow-up study
}

JONGHA LEE1, Hojun Lee1, Jihyun Kim2, Sangil Suh3, Moon-Soo Lee1

1Divison of child and adolescent psychiatry, Department of psychiatry, College of Medicine, Korea University 2Department of neurology, College of Medicine, Korea University 3Department of radiology, College of Medicine, Korea University

\section{Purpose}

This study aimed to compare the difference of structural connectivity between MDD patients and healthy control $(\mathrm{HC})$ by using Diffusion Tensor Imaging (DTI). We also investigated the change of structural connectivity in the patients between pretreatment and 3-month follow-up posttreatment.

\section{Method}

2.1 Participants: A total of 61 participants (34 patients and 27 HC) aged 13-19, who were diagnosed with MDD using DSM-4 TR by child psychiatrists, were recruited in the Korea University Guro hospital. We also gathered HCs who have never been treated in psychiatry clinics from local community and highschool. We have only recruited drug-naïve patient to exclude the effect of medications to brain structures.

2.2 Clinical measures: Every participant took intelligence test and we excluded participants who scored IQ under 85. The Korean version of investigator-rated Kiddies-SADS-Present and Lifetime Version (K-SADS-PL) was applied to confirm the diagnosis in all of the participants. Subject symptom information was gathered by using the Hamilton depression rating scale (HAM-D) and children's depression inventory (CDI) in all the participants including healthy controls.

2.3. Image data acquisition: $M R I$ scans were conducted with a 3 Tesla Siemens Skyra magnetic resonance system. Fractional anisotropy (FA), radial (RD), mean (MD), and axial diffusivity (AD) were estimated using DTI.

2.4 Statistical analysis: FMRIB Software Library (FSL) 5.0.10 and Tract Based Spatial Statistics (TBSS) were used for statistical analysis. IQ, duration of untreated period and HAM-D scores were included as covariate. Statistical analysis of clinical data was conducted using SPSS software, version 20.0.

Table 2. Multiple clusters where FA was increased and RD was reduced (comparison between the patient and the HCs)

\begin{tabular}{|c|c|c|c|c|c|c|c|}
\hline & \multirow[t]{2}{*}{$\begin{array}{c}\text { Cluster } \\
\text { No }\end{array}$} & \multirow[t]{2}{*}{$\begin{array}{c}\text { White matter } \\
\text { tract }\end{array}$} & \multicolumn{3}{|c|}{$\begin{array}{c}\text { MNI } \\
\text { coordinates } \\
\text { at signal peak }\end{array}$} & \multirow[t]{2}{*}{$\begin{array}{l}\text { Number } \\
\text { of } \\
\text { voxels }\end{array}$} & \multirow[t]{2}{*}{$\begin{array}{l}\text { Corrected } \\
P \text { value }\end{array}$} \\
\hline & & & $\mathbf{x}$ & $\mathbf{y}$ & $\mathbf{z}$ & & \\
\hline \multirow{4}{*}{$\begin{array}{c}\text { FA } \\
\uparrow\end{array}$} & 1 & Lt. $\operatorname{ATR}(63 \%)^{*}$ & -9 & -13 & 6 & 12 & 0.03 \\
\hline & 2 & Lt. $\operatorname{ATR}(47 \%)^{*}$ & -4 & -13 & 4 & 60 & 0.03 \\
\hline & 3 & Rt. CT $(11 \%)^{*}$ & 11 & -14 & -16 & 71 & 0.03 \\
\hline & 4 & Rt. ATR $(8 \%)^{*}$ & 4 & -24 & -17 & 257 & 0.03 \\
\hline \multirow{4}{*}{$\begin{array}{c}\text { RD } \\
\downarrow\end{array}$} & 4 & Lt. CT(58\%) & -21 & -18 & -3 & 102 & 0.02 \\
\hline & 5 & Lt. UF $(22 \%)^{*}$ & -34 & 0 & -15 & 181 & 0.02 \\
\hline & 6 & $\begin{array}{l}\text { Lt. ILF(18\%)* } \\
\text { Lt. SLF(5\%) }\end{array}$ & -41 & -17 & -20 & 251 & 0.02 \\
\hline & 7 & $\begin{array}{l}\text { Lt. IFOF }(8 \%)^{*} \\
\text { Lt. ILF }(5 \%)^{*}\end{array}$ & -10 & -88 & -6 & 254 & 0.02 \\
\hline
\end{tabular}

\section{Results}

1. Comparison between the patient and the HCs

1) FA was increased in both anterior thalamic radiation and Rt. corticospinal tract in MDD patients.

2) RD was reduced in multiple clusters near the both corticospinal tract, and superior and inferior longitudinal fasciculus in MDD patients.

\section{Comparison between pretreatment and 3-month follow-up}

1) $M D$ and $A D$ were increased statistically in multiple clusters after 3-month follow-up in patients group.

\section{Conclusion}

After 3-month pharmacotherapy, $M D$ and $A D$ were increased in corpus callosum and right superior corona radiate. The results were inconsistent with previous studies of adult depressive disorders. Increased FA in adolescents with MDD seems to be the risk factor for the development of depressive disorder.

Table 1. Demographical and clinical comparison between pretreatment and 3-month follow-up $(\mathrm{N}=23)$

\begin{tabular}{|c|c|c|c|c|}
\hline & \multirow{2}{*}{ Baseline } & \multirow{2}{*}{$\begin{array}{l}\text { 3-month } \\
\text { follow-up }\end{array}$} & \multicolumn{2}{|c|}{ Statistics } \\
\hline & & & $\mathbf{t}$ & $\mathbf{p}$ \\
\hline Female, n (\%) & \multicolumn{2}{|c|}{$14(60.86)$} & & \\
\hline Age, Years (SD) & \multicolumn{2}{|c|}{$15.48 \pm 1.76$} & \multicolumn{2}{|c|}{$(\operatorname{Min} 13, \operatorname{Max} 19)$} \\
\hline School Years (SD) & \multicolumn{2}{|c|}{$9.83 \pm 1.83$} & \multicolumn{2}{|c|}{$(\operatorname{Min} 7, \operatorname{Max} 13)$} \\
\hline IQ (SD) & \multicolumn{2}{|c|}{$102.43 \pm 12.45$} & \multicolumn{2}{|c|}{$(\operatorname{Min} 85, \operatorname{Max} 125)$} \\
\hline DUP, months (SD) & \multicolumn{2}{|c|}{$20.04 \pm 21.47$} & \multicolumn{2}{|c|}{$(\operatorname{Min} 1, \operatorname{Max} 75)$} \\
\hline CDI (SD) & $\begin{array}{r}27.96 \\
\pm 8.84 \\
\end{array}$ & $\begin{array}{r}18.13 \\
\pm 8.83 \\
\end{array}$ & 3.835 & $<.01$ \\
\hline HAM_D (SD) & $\begin{array}{r}19.65 \\
\pm 5.72\end{array}$ & $\begin{array}{r}13.52 \\
\pm 6.24\end{array}$ & 3.121 & $<.01$ \\
\hline
\end{tabular}

Table 3. Multiple clusters where AD and MD were increased (comparison between pretreatment and 3-month follow-up )

\begin{tabular}{|c|c|c|c|c|c|c|c|}
\hline & \multirow[t]{2}{*}{$\begin{array}{c}\text { Cluster } \\
\text { No }\end{array}$} & \multirow[t]{2}{*}{$\begin{array}{c}\text { White matter } \\
\text { tract }\end{array}$} & \multicolumn{3}{|c|}{$\begin{array}{c}\text { MNI } \\
\text { coordinates } \\
\text { at signal peak }\end{array}$} & \multirow{2}{*}{$\begin{array}{c}\text { Number } \\
\text { of } \\
\text { voxels }\end{array}$} & \multirow[t]{2}{*}{$\begin{array}{l}\text { Corrected } \\
\mathbf{P} \text { value }\end{array}$} \\
\hline & & & $\mathbf{x}$ & $y$ & z & & \\
\hline \multirow{3}{*}{$\begin{array}{c}\text { AD } \\
\uparrow\end{array}$} & 1 & Body of $\mathrm{CC}^{* *}$ & 8 & -11 & 27 & 1278 & 0.03 \\
\hline & 2 & Genu of $\mathrm{CC}^{* \star}$ & 9 & 26 & 13 & 137 & 0.05 \\
\hline & 3 & $\begin{array}{l}\text { Rt. CT }(11 \%)^{*} \\
\text { Rt. SLF }(3 \%)^{*}\end{array}$ & 28 & -12 & 25 & 13 & 0.05 \\
\hline \multirow{3}{*}{$\begin{array}{c}\text { MD } \\
\uparrow\end{array}$} & 1 & $\begin{array}{l}\begin{array}{l}\text { Splenium of } \\
\text { CC ** }\end{array} \\
\text { Lt. ATR }(3 \%)^{*}\end{array}$ & -14 & -34 & 27 & 3609 & 0.02 \\
\hline & 2 & $\begin{array}{c}\text { Rt. SCR } \\
\text { Rt. CT }(24 \%)^{\star}\end{array}$ & 27 & -16 & 28 & 1256 & 0.02 \\
\hline & 3 & Rt. SLF $(11 \%)^{*}$ & 31 & 5 & 16 & 2 & 0.03 \\
\hline
\end{tabular}

ATR = Anterior thalamic radiation, CT = Corticospinal tract, IFOF = Inferior fronto-occipital fasciculus, IFL $=$ Inferior longitudinal fasciculus, SLF $=$ Superior longitudinal fasciculus, SCR = Superior corona radiata, UF = Uncinate fasciculus, JHU White-matter Tractography *, JHU ICBM-DTI-81 White-Matter Labels * 